\title{
Building extraction based on OpenStreetMap tags and very high spatial resolution image in urban area
}

\author{
L. Kang ${ }^{1,2}$, Q. Wang ${ }^{3}$, H. W. Yan ${ }^{1,2, *}$ \\ ${ }^{1}$ Faculty of Geomatics, Lanzhou Jiaotong University, Lanzhou 730070, China - (kanglu19920831, wangqing.cugb, \\ haowen2010)@gmail.com \\ ${ }^{2}$ Gansu Provincial Engineering Laboratory for National Geographic State Monitoring, Lanzhou 730070, China \\ ${ }^{3}$ School of the Earth Sciences and Resources, China University of Geosciences, Beijing 100083, China
}

KEY WORDS: Building extraction, OpenStreetMap, Very High Spatial Resolution, Tag-based, Automatic interpretation, urban area

\begin{abstract}
:
How to derive contour of buildings from VHR images is the essential problem for automatic building extraction in urban area. To solve this problem, OSM data is introduced to offer vector contour information of buildings which is hard to get from VHR images. First, we import OSM data into database. The line string data of OSM with tags of building, amenity, office etc. are selected and combined into completed contours; Second, the accuracy of contours of buildings is confirmed by comparing with the real buildings in Google Earth; Third, maximum likelihood classification is conducted with the confirmed building contours, and the result demonstrates that the proposed approach is effective and accurate. The approach offers a new way for automatic interpretation of VHR images.
\end{abstract}

\section{INTRODUCTION}

Buildings including houses, bridges, roads and large-scale engineering buildings, which are various forms of places where people gather and settle for the needs of production and living. As a major artificial object closely related to human life, building is an important symbol of urban development and one of the most important research subjects in urban problems. Information of buildings can be used to study the expansion of cities, urban planning, urban heat island effect, 3D city modelling, geographic information systems (GIS) database updating, etc. (Wu et al., 2005). With the development of aeronautics and astronautics, the spatial resolution of remote sensing images is increasing and the difficulty in obtaining remote sensing data is reducing. Thematic information extraction from very high spatial resolution (VHR) remote sensing images has attracted more and more scholars' attention (Stefanov et al., 2001) and building extraction from VHR remote sensing images has become an important mean of building information acquisition. Although VHR images have more details, it brings specific problems like shades that normal images cannot illustrate (Carleer et al., 2005, Tan et al., 2010). How to derive buildings from VHR images automatically and precisely is a challenging problem needing to be solved (Du and Zhang, 2014, Huang and Zhang, 2013, Moser et al., 2013, Zhang et al., 2012, 2013).

From the perspective of extraction methods, existing work generally falls into edge-based geometric grouping or objectbased classification (Du et al., 2015). The former method is limited by the accuracy of the contour extracted from the image and the complexity of the image (Croitoru \& Doytsher, 2004). The other kind of method, object-based methods first segment VHR images into objects, and then distinguish the objects with characteristics of buildings from all objects (Myint et al., 2011).

Since Goodchild first proposed the concept of Volunteered Geographic Information (VGI) in 2007 (Goodchild, 2007). VGI has been developing prosperously as a newly emerged geoinformation industry. The OpenStreetMap (OSM) as one of the most famous VGI projects has generated a world map contributed from millions of contributors within an open source environment during the last decade. Theoretically everyone can use or edit OpenStreetMap data without restrictions. OpenStreetMap represents physical features on the ground (e.g., roads or buildings) using tags attached to its basic data structures (its nodes, ways, and relations). Each tag describes a geographic attribute of the feature being shown by that specific node, way or relation. OSM's free tagging system allows the map to include an unlimited number of attributes describing each feature. The community agrees on certain key and value combinations for the most commonly used tags, which act as informal standards. These commonly used tags include buildings, highways etc. can be extracted from OSM data and have potential to provide a more detailed building extraction result.

In this study, OSM data was introduced to extract contour information of buildings. Using the contour extracted as training samples, the buildings in the whole VHR image could be extracted automatically. Experimental results indicate that this building extraction method is useful for automatic interpretation of VHR remote sensing images. The data sources of this study are discussed in Section 2.1. The methodology, introducing OSM tags-based building contour extraction and how to combine OSM extraction results and remote sensing images are presented in Sections 2.2 and 2.3, respectively. The experimental results and discussion are described in Section 3. Final conclusions are presented in Section 4. 


\section{METHODOLOGY}
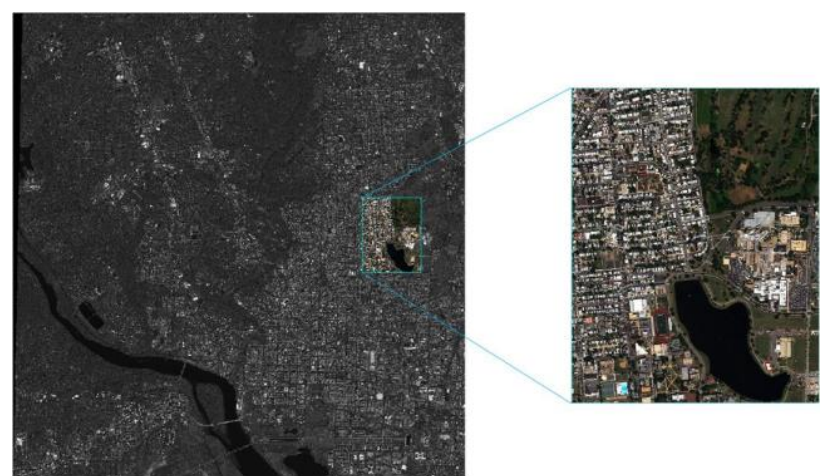

Figure 1. The study area

Firstly, the contour of buildings (Vegetation and water area are also extracted to perform supervised classification) was extracted using OSM tags. Following this, in the study site of Washington D.C (Figure 1), maximum likelihood classification of the VHR remote sensing image was conducted (Figure 2).

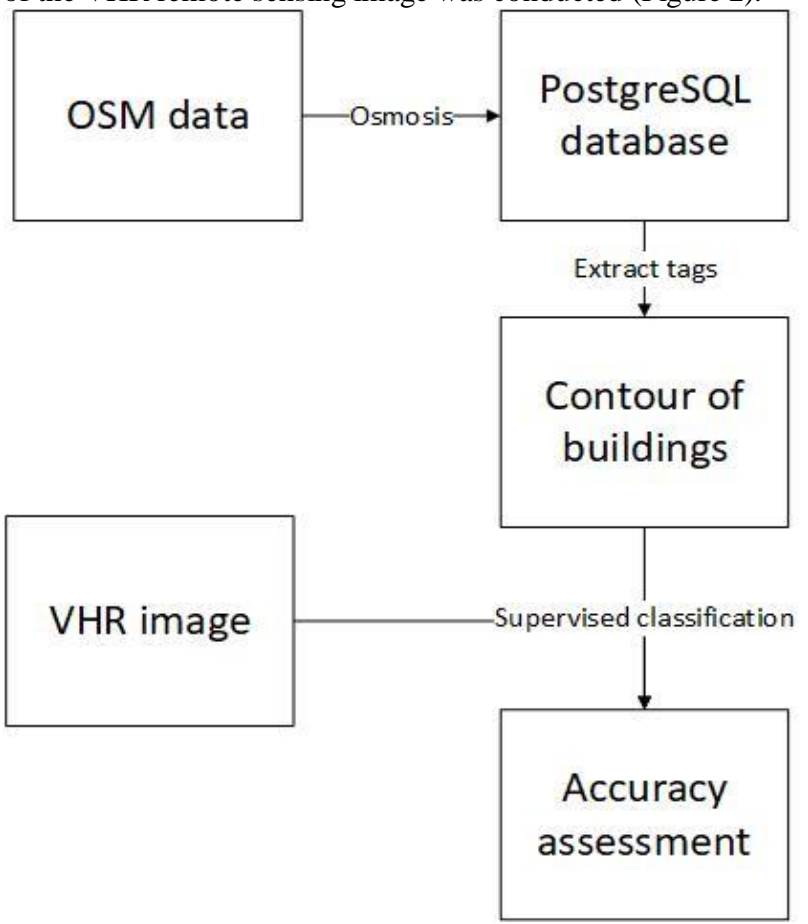

Figure 2. Workflow of the study method

\subsection{Data sources}

OpenStreetMap is a freely-licensed, global geospatial database. It provides a weekly-updated dataset at global scale. The OSM vector file of Washington D.C was downloaded on the 2nd of January, 2018.

WorldView-2, launched October 2009, is the first highresolution 8-band multispectral commercial satellite. Operating at an altitude of $770 \mathrm{~km}$, WorldView-2 provides $46 \mathrm{~cm}$ panchromatic resolution and $1.85 \mathrm{~m}$ multispectral resolution. WorldView-2 Map Ready products of Washington D.C was choosing to be the VHR remote sensing images source.

\subsection{Extract Vector Contours of Buildings from OSM tags}

In the first place, we create a new PostgreSQL database with hstore extension and a database-schema for storing OSM data. Then we use osmosis (a command line application for processing OSM data) to import OSM file into the database we created previously. Following this, SQL scripts are applied to create a SQL view containing rows with specific tags like building, amenity or office etc. (Goetz et al., 2012). Then we can load the SQL view by connecting the database in QGIS. Finally the extraction output is as shown in the Figure 3 and the extraction result was convert to $\mathrm{kml}$ file and loaded in Google Earth to further confirm its accuracy. The extraction result was pretty good compare to the Google Earth.

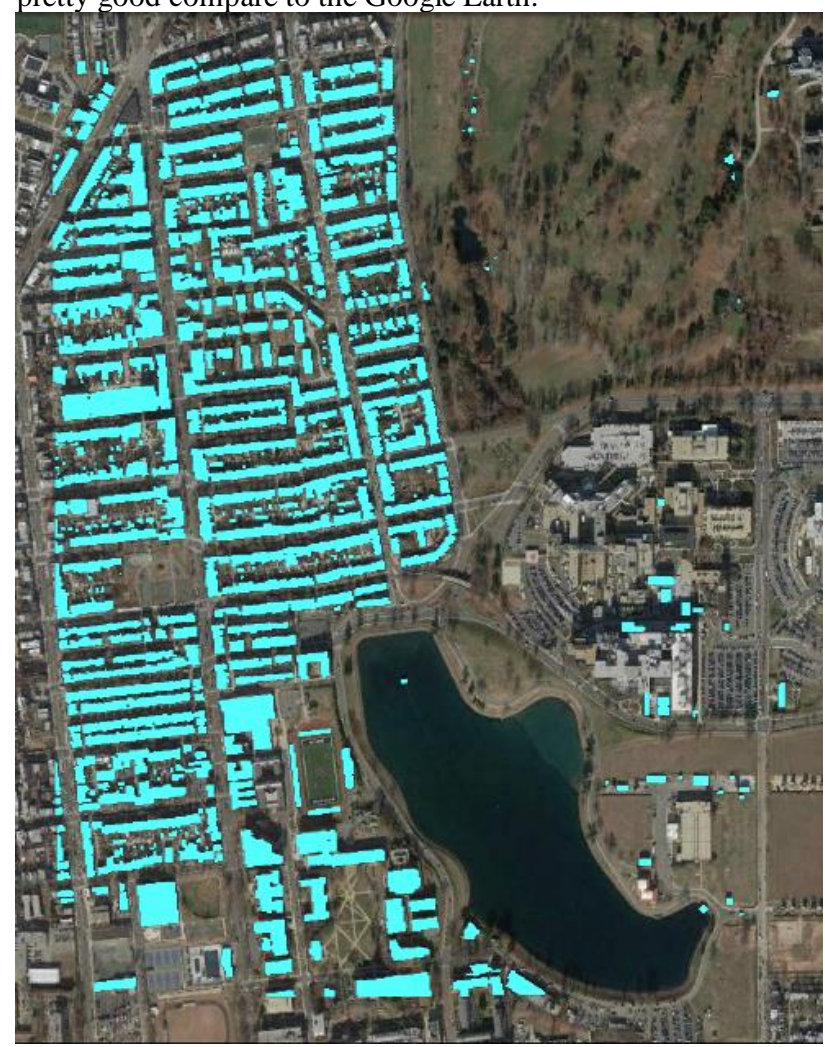

Figure 3. Builds extracted from Open Street Map and the comparison with Google Earth

\subsection{Combine VHR Image with OSM Contour and the supervised classification}

As we can see from the metadata, the coordinate system of the image is WGS 84. Since OSM data are also stored as coordinates according to WGS 84 , it is quite easy for us to load contours extracted from OSM data and the remote sensing images at the same time.

After that, water and vegetation in the study area were extracted with the same method to conduct supervised classification. To balance the number of samples for buildings, water and vegetation, we selected dozens of samples randomly for a better classification. The samples for classification were as shown in Figure 4 and the classification result was shown in Figure 5. 


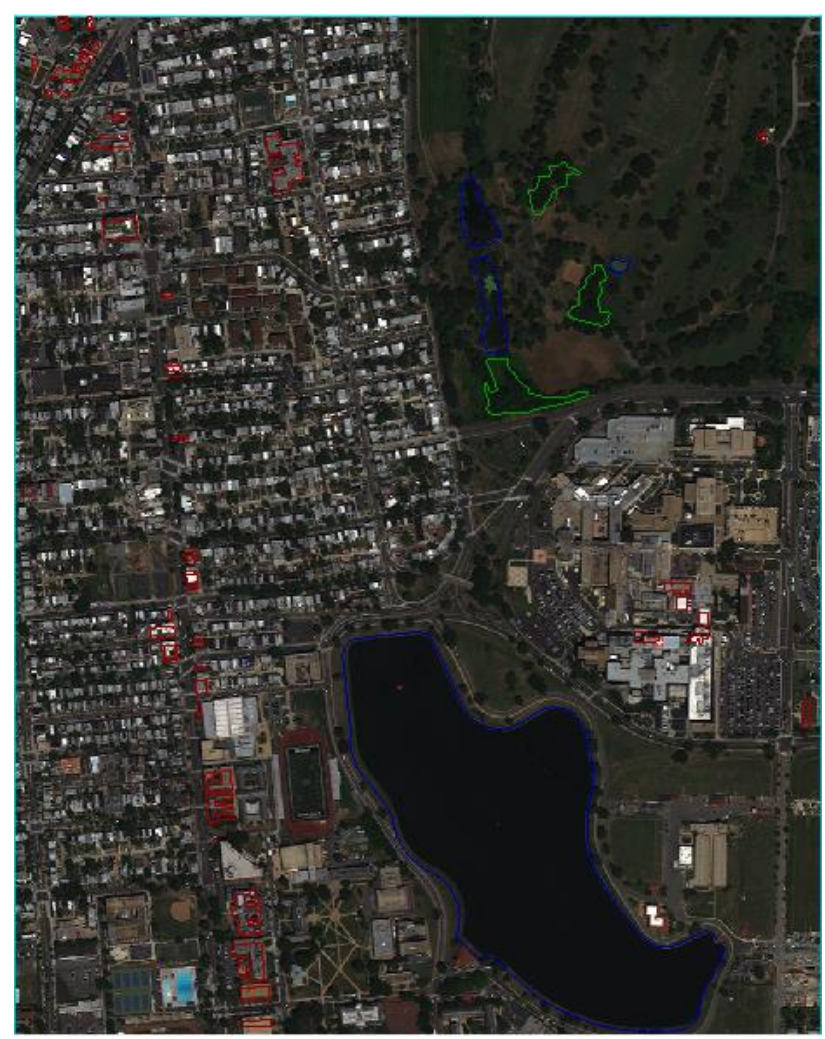

Figure 4. Samples for supervised classification loaded with VHR image

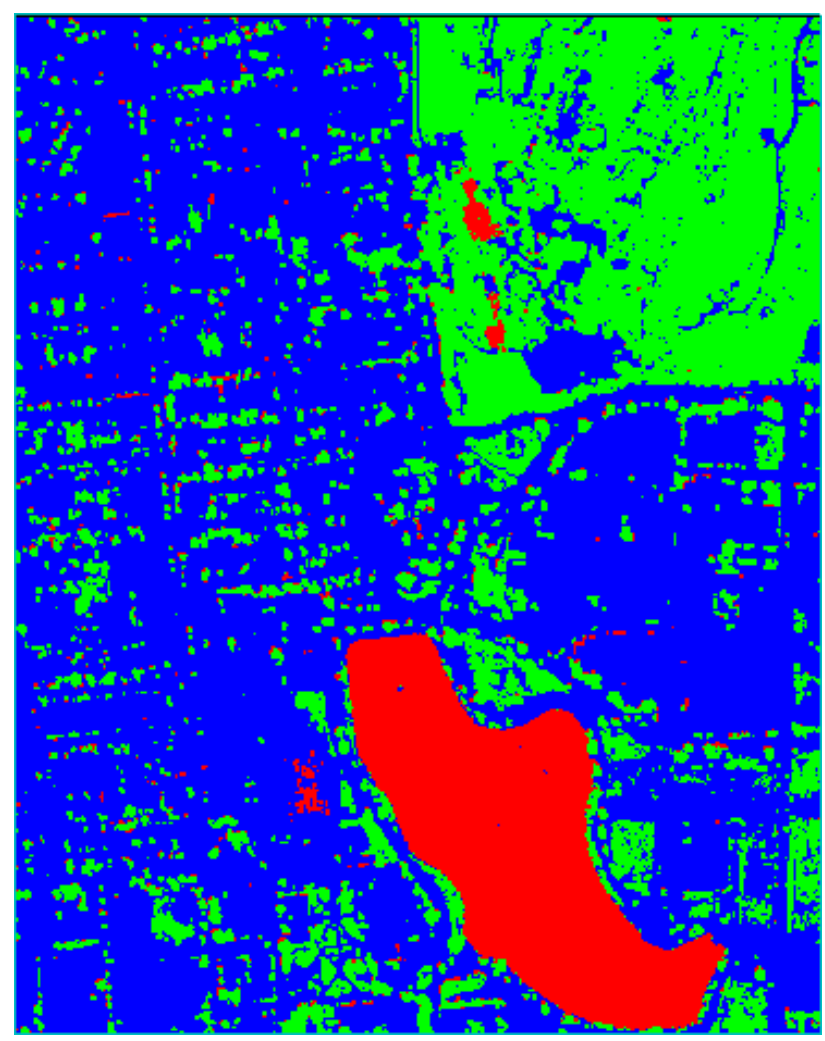

Figure 5. Classification result of maximum likelihood method

We use maximum likelihood classification method to classify the selected image. Using all buildings, vegetation and water extracted from OSM as ground truth ROI and the confusion matrix shows that user's accuracy of the maximum likelihood classification is $99.1 \%$, producer's accuracy is $86.8 \%$. The classification result is good with few randomly selected samples.

\section{DISCUSSION}

Some challenges occur while trying to use voluntarily contributed OSM data. 1. Incorrect tags or absent tags. OSM tags are added by individual users rather than specialists, users cannot recognize every feature they meet while trying to add their own GPS tracks and tags are not pre-requisite to upload OSM data; 2. Features updated at different time. Since users can contribute upload data at any time, data stored in OSM database is not updated at the same time. Although OSM database is updated every 7 days on a planetary scale, not every single data raw is changed. Some features haven't been changed since 2007 while others are up to date. 3. Incomplete area-coverage of OSM data. The amount of OSM data is abundant in Europe, but it is incomplete in Asia, South America and Africa in contrast. For example, the amount of OSM data of Europe is 20GB in the meantime the world's data is $38 \mathrm{~GB}$. Europe that covers only $6.8 \%$ the Earth's land area takes up almost $50 \%$ of the OSM database.

Despite these challenges above, OSM can provide a more detailed and accurate representation of buildings than that achieved solely through VHR remote sensing imagery. Some scholars have used OSM to extract the land cover classification map (Schultz et al., 2017) and use the OSM label to update existing land cover products (Fonte et al., 2017). But they are limited by minimum mapping unit (MMU) of remote sensing images. Spatial resolution of VHR images are at decimetre level. By using this kind of images restrictions of MMU can be reduced to minimum.

\subsection{Why tags from OSM can be used to extract buildings?}

First, OSM's vector data structure is better to describe geological objects rather than raster images. And buildings' contour was extracted by connecting the nodes according to their relations. As for our study site, we extracted 160 thousand of buildings and almost every building's profile could fit its contour in google earth. Thus, we conclude that it is possible to extract buildings with OSM tags.

\subsection{How to extract buildings in the areas with little OSM data?}

The Inhomogeneous distribution of OSM data leads to the problem that in some area, like rural areas, where less people are likely to contribute to the map. Since there are still buildings can be extracted in these areas, we can take them as training samples for supervised classification of VHR images. This method can combine morphological character from OSM and remote sensing images.

\section{CONCLUSION}

In this study, we tried to extract building information from OSM database with tags using comprehensive SQL language. Washington D.C. where OSM data is rich enough to directly extract almost all the buildings using OSM tags was chose as research area and the accuracy of the data source is confirmed by comparing the extraction result to google earth. Some 
buildings, vegetation, water area from the OSM extraction are selected randomly as the training samples for VHR remote sensing image (WorldView-2 Map Ready products of Washington D.C.). Maximum likelihood classification method was conducted to classify the selected image and the classification accuracy appears good. The result shows that building extraction by OSM tags is far more efficient than traditional ways like distinguish buildings manually using VHR images. For some area, buildings can be extracted by tags precisely; for most area, in-complete OSM data can serve as training samples for supervised classification by remote sensing result in a good accuracy.

\section{ACKNOWLEDGEMENTS}

Work described in this paper is supported by National Key R\&D Program of China project under Grant No. 2017 YFB0504203 and the National Science Foundation of China project under Grant No. 41671447.

\section{REFERENCES}

Carleer, A. P., Debeir, O., \& Wolff, E. 2004. Comparison of very high spatial resolution satellite image segmentations. In Image and Signal Processing for Remote Sensing IX (Vol. 5238, pp. 532-543). International Society for Optics and Photonics.

Croitoru, A., \& Doytsher, Y. 2004. Right - Angle Rooftop Polygon Extraction in Regularised Urban Areas: Cutting the Corners. The Photogrammetric Record, 19(108), 311-341.

Du, B., \& Zhang, L. 2014. A discriminative metric learning based anomaly detection method. IEEE Transactions on Geoscience and Remote Sensing, 52(11), 6844-6857.

Du, S., Zhang, F., \& Zhang, X. 2015. Semantic classification of urban buildings combining VHR image and GIS data: An improved random forest approach. ISPRS journal of photogrammetry and remote sensing, 105, 107-119.

Fonte, C. C., Minghini, M., Patriarca, J., Antoniou, V., See, L., \& Skopeliti, A. 2017. Generating up-to-date and detailed land use and land cover maps using OpenStreetMap and GlobeLand30. ISPRS International Journal of Geo-Information, 6(4), 125.

Goetz, M., Lauer, J., \& Auer, M. 2012. An algorithm based methodology for the creation of a regularly updated global online map derived from volunteered geographic information. In Proceedings of the Fourth International Conference on Advanced Geographic Information Systems, Applications, and Services, Valencia, Spain (Vol. 30, pp. 50-58).

Goodchild, M. F. 2007. Citizens as sensors: the world of volunteered geography. GeoJournal, 69(4), 211-221.

Huang, X., \& Zhang, L. 2013. An SVM ensemble approach combining spectral, structural, and semantic features for the classification of high-resolution remotely sensed imagery. IEEE transactions on geoscience and remote sensing, 51(1), 257-272.

Moser, G., Serpico, S. B., \& Benediktsson, J. A. 2013. Landcover mapping by Markov modeling of spatial-contextual information in very-high-resolution remote sensing images. Proceedings of the IEEE, 101(3), 631-651.

Myint, S. W., Gober, P., Brazel, A., Grossman-Clarke, S., \& Weng, Q. 2011. Per-pixel vs. object-based classification of urban land cover extraction using high spatial resolution imagery. Remote sensing of environment, 115(5), 1145-1161.

Schultz, M., Voss, J., Auer, M., Carter, S., \& Zipf, A. 2017. Open land cover from OpenStreetMap and remote sensing. International Journal of Applied Earth Observation and Geoinformation, 63, 206-213.

Stefanov, W. L., Ramsey, M. S., \& Christensen, P. R. 2001. Monitoring urban land cover change: An expert system approach to land cover classification of semiarid to arid urban centers. Remote sensing of Environment, 77(2), 173-185.

Tan, Q., Wei, Q., \& Liang, F. 2010. Building extraction from VHR multi-spectral images using rule-based object-oriented method: A case study. In Geoscience and Remote Sensing Symposium (IGARSS), 2010 IEEE International (pp. 27542756). IEEE.

Wu, S. S., Qiu, X., \& Wang, L. 2005. Population estimation methods in GIS and remote sensing: a review. GIScience \& Remote Sensing, 42(1), 80-96.

Zhang, L., Zhang, L., Tao, D., \& Huang, X. 2012. On combining multiple features for hyperspectral remote sensing image classification. IEEE Transactions on Geoscience and Remote Sensing, 50(3), 879-893.

Zhang, L., Zhang, L., Tao, D., \& Huang, X. 2013. Tensor discriminative locality alignment for hyperspectral image spectral-spatial feature extraction. IEEE Transactions on Geoscience and Remote Sensing, 51(1), 242-256. 